International Journal of Modern Physics B

(C) World Scientific Publishing Company

\title{
ELECTRIC CURRENT GENERATION IN DISTORTED GRAPHENE
}

\author{
ANA JULIA MIZHER \\ Instituto de Ciencias Nucleares, Universidad Nacional Autónoma de México \\ Apartado Postal 70-543, México Distrito Federal 04510, Mexico \\ ALFREDO RAYA \\ Instituto de Física y Matemáticas, Universidad Michoacana de San Nicolás de Hidalgo \\ Edificio C-3, Ciudad Universitaria, C.P. 58040, Morelia, Michoacán, Mexico \\ CRISTIÁN VILLAVICENCIO \\ Departamento de Ciencias Básicas, Universidad del Bío-Bío \\ Casilla 447, Chillán, Chile
}

\begin{abstract}
Graphene-like materials can be effectively described by Quantum Electrodynamics in $(2+1)$-dimensions. In a pristine state, these systems exhibit a symmetry between the nonequivalent Dirac points in the honeycomb lattice. Realistic samples which include distortions and crystaline anisotropies are considered through mass gaps of topological and dynamical nature. In this work we show that the incorporation of an in-plane uniform external magnetic field on this pseudochiral asymmetric configuration generates a nondissipative electric current aligned with the magnetic field: The pseudo chiral magnetic effect. This scenario resembles the chiral magnetic effect in Quantum Chromodynamics.

Keywords: Graphene; Magnetic field; Chiral magnetic effect
\end{abstract}

\section{Introduction}

In less than a decade from the emergence of the so-called Dirac and Weyl Materials -among which graphene ${ }^{1,2}$ and more recently, topological insulators ${ }^{3}$, have attracted considerable attention, a renewed interest within both the elementary particle and condensed matter physics has developed around the behavior of planar fermions, which have transited from being toy models of Quantum Chromodynamics (QCD) under extreme conditions to actual players of a revolutionary era for fundamental physics and technological application prospects.

Graphene and related materials are composed by a single layer of atoms tightly packed into a two-dimensional honeycomb array and therefore can be efficiently described by tight-binding models. In the continuum limit, these models can be mapped into the Hamiltonian of $(2+1)$-dimensional quantum electrodynamics $\left(\mathrm{QED}_{3}\right)$ with massless Dirac fermions. ${ }^{2}$ A variety of traditional condensed matter 
phenomena found an effective description in terms of the $\mathrm{QED}_{3}$ degrees of freedom, including high- $T_{c}$ superconductivity ${ }^{4}$ and quantum Hall effect. ${ }^{5}$ It was, however, the gapless nature of the charge carriers in graphene at low energy, which around the Dirac points of the Brillouin zone of the honeycomb lattice exhibit a linear dispersion relation what boosted the interest on the properties of "relativistic" planar fermions in a condensed matter environment.

Although being Abelian, $\mathrm{QED}_{3}$ exhibits similar features to non-Abelian gauge theories, hence establishing a link between particle physics and condensed matter systems. Therefore, $\mathrm{QED}_{3}$ opens the possibility to explore phenomena which are either inaccessible, due to energy limitations, or hard to measure in a particle physics experiment. Indeed, it is known that at very high temperatures, a non-Abelian gauge theory coupled to $N_{f}$ fermion families in $(3+1)$-space-time dimensions experiences a dimensional reduction to an effective $(2+1)$-dimensional theory, which further "abelianizes" if $N_{f}$ is large enough ${ }^{6}$; non-abelian interactions are suppressed by a factor of $N_{f}^{-1}$. This fact makes $\mathrm{QED}_{3}$ an effective version of QCD, which also exhibits important non-perturbative phenomena like confinement and dynamical chiral symmetry breaking. ${ }^{7}$ In particular, in the same way as it happens in QCD, the Lagrangian of $\mathrm{QED}_{3}$ admits a non-trivial Chern-Simons (CS) term ${ }^{8}$ which manifests itself as a gauge boson mass of topological nature, thus allowing for the possibility of time reversal and (generalized) parity breaking, fractional statistics and so on (see, for instance, Ref. 9).

In this work we propose that, for some planar systems effectively described by $\mathrm{QED}_{3}$ in the presence of an external in-plane magnetic field, it occurs a mechanism which manifests itself as the generation of a non-dissipative electric current along the direction of the magnetic field. Such a current has a topological nature, and may be regarded as the analogue for a bi-dimensional system of an effect proposed in the context of the quark gluon plasma produced in heavy ion collisions, known as chiral magnetic effect (CME) ${ }^{10}$ The effect we describe is not related with the spin of charge carriers, but pseudospin. We refer to it as pseudochiral magnetic effect (PCME).

\section{The PCME in graphene}

The CME is characterized by the interaction between the topological gauge fields and the fermions (in this case, deconfined quarks), that causes a flip in the chirality of the latter, generating domains of homogeneous chirality. Because in $(3+1)$ dimensions, chirality in the massless limit corresponds to helicity, a relation between the directions of spin and momentum is established, and in the presence of an external uniform magnetic field, the magnetic alignment of spins the spins with it generates an electric current in the field direction.

In the massless Dirac theory, where $\gamma^{5}$ commutes with the Hamiltonian, the chirality quantum number is a conserved quantity and it is possible to define a representation where the spinors that describe the quasiparticle excitations are eigen- 
states of $\gamma^{5}$. In $(2+1)$-dimensions it is not possible to perform rotations around the direction of the momentum and the spin operator loses its usual physical meaning. In other words, the concept of helicity related to Lorentz group and real space rotations is meaningless. However, one can still construct an operator that commutes with the Hamiltonian and has the chiral conserved quantum number as its eigenvalue. In analogy with the spin in (3+1)-dimensions, this is called pseudospin operator and corresponds to an internal symmetry rather than to spatial symmetry. ${ }^{2}$ In this case, the eigenstates of the $\gamma^{5}$ operator are the spinors that, in the case of monolayer graphene, represent the quasiparticle excitation at the two inequivalent Dirac points in the first Brillouin zone of the graphene honeycomb, $K_{+}$and $K_{-}$, and therefore the pseudohelicity can be seen as a flavor label corresponding to each one of the Dirac points. Considering the prescription described above, a breaking of the pseudohelicity symmetry in planar systems corresponds to an imbalance between the inequivalent Dirac points, which can be, for instance, by generating different effective masses for each one of them.

Electrons in crystal lattices behave like quasiparticles, which means that their interactions can be represented by effective masses. Generally speaking, in graphenelike materials, beyond the free electron picture, masses or gaps can be opened through a variety of external perturbations, e.g. strong enough magnetic fields, but also through mechanical distortion of the underlying lattice structure. ${ }^{11}$ A total of 36 gap-opening instabilities of the Dirac type in the spin, valley and superconducting channels have been considered in graphene and graphene-like structures (see Ref. 12 and references therein). To establish the pseudohelicity symmetry breaking, we are particularly interested in mechanisms that generate different masses for the inequivalent Dirac points. A physical realization of this prescription is given in Ref. 13, where the authors propose to place the graphene membrane over a hexagonal boron nitride layer, that is conformed in such a way that its lattice coincides with the graphene honeycomb lattice, generating a different effective mass for the charge carriers from different points $K_{+}$and $K_{-}$. Such an inequivalence has proven to be valid, for instance, for strained graphene, where pseudomagnetic fields are generated in terms of effective masses ${ }^{14}$. In fact, the effect of intrinsic curvature of the graphene membrane at the level of the equations of motion of charge carriers can be considered as if these particles develop an effective masses in flat space ${ }^{15}$. In the same way, in this work we consider a generalized deformed graphene layer aligned with a magnetic field permeating it. Schematically shown in Fig. 1, the deformations and distortions of the honeycomb lattice can be described as a perfectly planar system by the inclusion of Haldane masses.

Representing the pseudohelicity breaking in terms of a field theory, we look for a combination of masses in the Lagrangian, among all those allowed for such systems, that results in different masses for each eigenstate of the chiral operator. The particular choice of the masses proportional to $\gamma^{3}$ and $\gamma^{3} \gamma^{5}$ generates this pseudochirality imbalance, still keeping the Lagrangian invariant under the pseudochiral transfor- 


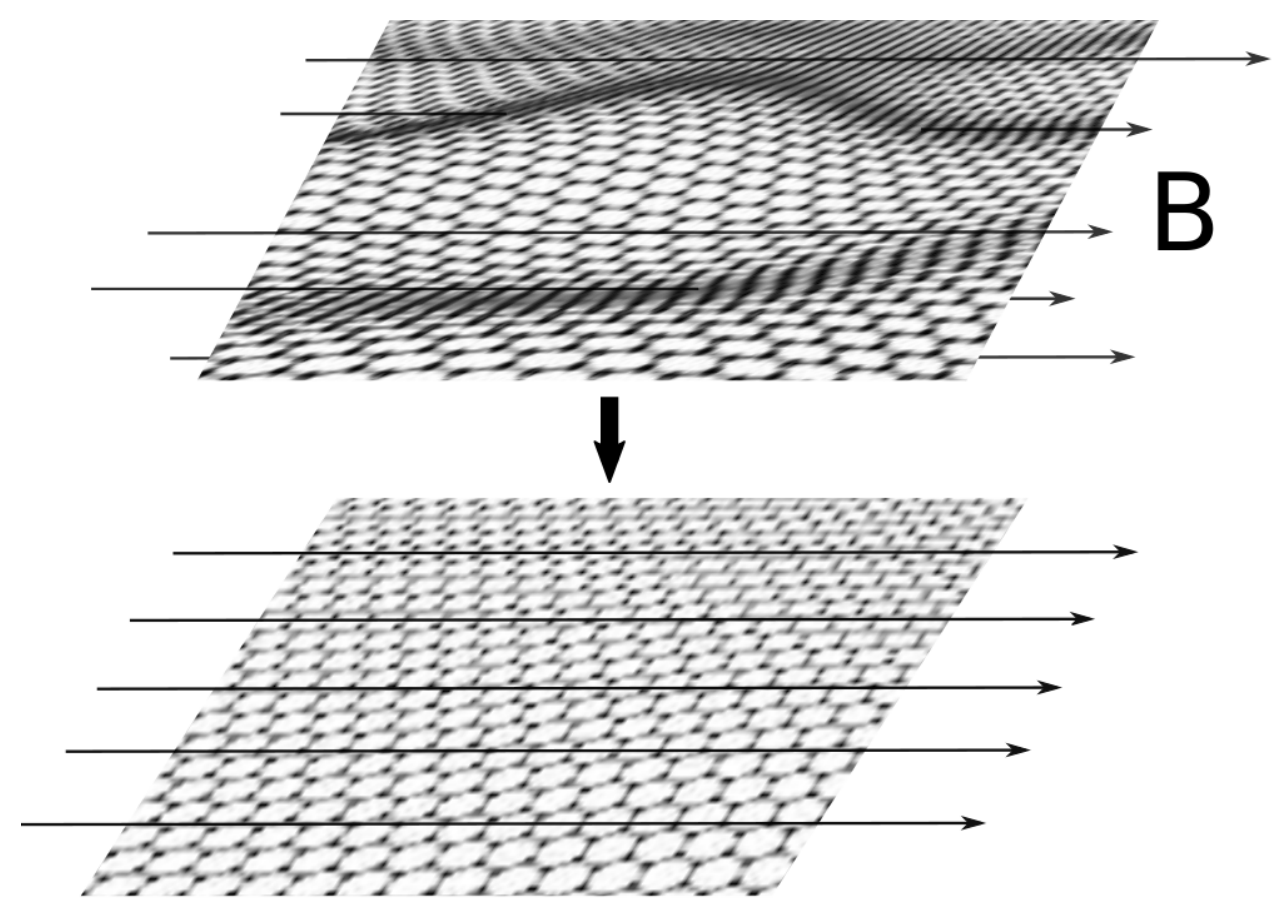

Fig. 1. A membrane of corrugated graphene in an in-plane magnetic field is idealized as a flake of pristine graphene where charge carriers from different sublattices have different effective masses.

mation $\psi \rightarrow e^{i \theta \gamma^{5}} \psi$. An interesting feature about the mass proportional to $\gamma^{3} \gamma^{5}$ is that it generates a CS term in the gauge sector of the Lagrangian, as well as a CS term generates this mass term in the fermion Lagrangian. ${ }^{16}$ Therefore, the chosen mass term structure reinforces our analogy with the CME in QCD, where the CS term is also responsible for the breaking of helicity symmetry.

\section{Lagrangian with planar magnetic field}

Completing the analogy, we require a Lagrangian that includes a magnetic field with field lines pointing along the graphene plane. Unlike the case of quantum Hall effect with a magnetic field perpendicular to the graphene sheet, the gauge field that generates this in-plane magnetic field can depend on the coordinate perpendicular to the plane. Besides brane-world scenarios, where gauge invariance is guaranteed by construction (see Ref. 17 and references therein), an appropriate treatment is to extend the theory to a (3+1)-dimensional space-time with a compactified dimension, and then perform a strict dimensional reduction. An extended dimensionality is a more realistic scenario in the sense that graphene indeed has an effective thickness besides the corrugations. However, if we want to consider the intersection of this external classical gauge field with the plane directly, there is no $\operatorname{SO}(1,3)$ gauge symmetry anymore, the $A_{3}^{\text {ext }}$ component decouples from the other gauge vector 
components and now the gauge components $A_{0}^{\text {ext }}, A_{1}^{\text {ext }}, A_{2}^{\text {ext }}$ are constrained to an $\mathrm{SO}(1,2)$ symmetry ${ }^{18}$.

This scenario is similar to what is observed in high temperature dimensional reduction, where the $A_{0}$ component decouples from the other gauge vector fields. ${ }^{19}$ The correct way to deal with this situation is to choose a gauge which does not depend on the perpendicular coordinate. In this case the dimensional reduction is guaranteed and therefore we can treat the system as a $(2+1)$-dimension one scketched in Fig. 1.

Considering an external magnetic field homogeneous in space and time, the only gauge component that generates the planar magnetic field is $A_{3}^{\text {ext }}$, since contribution from other vector components must depend on the dynamics of the transverse dimension. If the external magnetic field points in the $x$-direction, we choose the Landau-like gauge $A_{3}^{\text {ext }}=-B y$, where the curl of this gauge field generates the in-plane magnetic field and its coordinate dependence is also in the plane. ${ }^{\mathrm{a}}$ The other external electromagnetic components are still gauge invariant.

The resulting general chiral invariant Lagrangian, in Minkowski space, is:

$$
\mathcal{L}_{F}=\bar{\psi}\left[i \not D+\left(e A_{3}^{\mathrm{ext}}-m_{3}\right) \gamma^{3}-m_{o} \gamma^{3} \gamma^{5}\right] \psi
$$

where $D=\left(\partial_{0}-i \mu, v_{F} \boldsymbol{\nabla}\right), e$ is the fundamental charge, $v_{F}$ is the Fermi velocity and $\mu$ the chemical potential. The fields $\psi$ are 8 -component spinors which correspond to the direct product spin $\otimes$ pseudospin. As we do not include spin interactions, spin label will not be treated explicitly in what follows, but must be taken into account. For simplicity we will set $v_{F}=1$ or, in other words, $v_{F}$ will be the new speed of light. Notice that because we describe the propagation of negative charge-carriers (quasiparticles and not holes), the chemical potential must be positive.

Recalling the discussion in the previous section, the presence of a CS term automatically generates the mass $m_{o}$ in the fermion Lagrangian. This mass is referred to in literature as the odd mass ${ }^{16}$ regarding its parity non-preserving character. The mass term $m_{3}$ reproduces the asymmetry between the sub-lattices generated by, for example, placing the graphene membrane on top of a hexagonal boron nitride layer. ${ }^{13 \mathrm{~b}}$

\section{Green Function}

In the Weyl representation for the gamma matrices, the charge carriers Lagrangian can be separated in two chiralities:

$$
\mathcal{L}_{F}=\sum_{\chi= \pm} \bar{\psi}_{\chi}\left[i \not \partial+\mu \gamma^{0}+\left(e A_{3}^{\mathrm{ext}}-m_{\chi}\right) \gamma^{3}\right] \psi_{\chi}
$$

with the fields and masses defined as $\psi_{ \pm}=\frac{1}{2}\left(1 \pm \gamma^{5}\right) \psi$ and $m_{ \pm}=m_{3} \pm m_{o}$. The Green function in configuration space can be written in terms of the combination

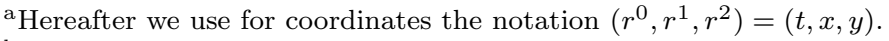

b In the Dirac representation, the mass $m_{3}$ corresponds to the standard Dirac mass. 
of the Green function of each chirality as

$$
G\left(r, r^{\prime}\right)=\frac{1}{2}\left(1+\gamma^{5}\right) G_{+}\left(r, r^{\prime}\right)+\frac{1}{2}\left(1-\gamma^{5}\right) G_{-}\left(r, r^{\prime}\right),
$$

where the chiral Green functions are defined as

$$
G_{ \pm}\left(r, r^{\prime}\right)=\left\langle r\left|\frac{i}{\not \Lambda+\left(e A_{3}^{\text {ext }}-m_{ \pm}\right) \gamma^{3}}\right| r^{\prime}\right\rangle
$$

with $\Pi=\left(i \partial_{0}+\mu, i \boldsymbol{\nabla}\right)$. Since the operators $\Pi_{0}$ and $\Pi_{1}$ commute with the other operators involved, $\Pi_{2}$ and $A_{3}^{\text {ext }}$, we can introduce a set of eigenstates $\left|k^{0}\right\rangle$ and $\left|k^{1}\right\rangle$. The chiral Green functions, then, can be written as

$$
G_{ \pm}\left(r, r^{\prime}\right)=\int \frac{d^{2} k_{\|}}{(2 \pi)^{3}} e^{-i k_{\|} \cdot\left(r-r^{\prime}\right)}\left\langle y\left|\frac{K_{\|}+\Pi_{2} \gamma^{2}+\left(e A_{3}^{\text {ext }}-m_{ \pm}\right) \gamma^{3}}{i H_{ \pm}}\right| y^{\prime}\right\rangle
$$

where we have introduced the Hamiltonian in the proper-time method, defined as

$$
H_{ \pm} \equiv-K_{\|}^{2}+\Pi_{2}^{2}+\left(e A_{3}^{\mathrm{ext}}-m_{ \pm}\right)^{2}+i e B \gamma^{2} \gamma^{3}
$$

and where the parallel momentum vectors are defined as $k_{\|}=\left(k^{0}, k^{1}, 0\right)$ and $K_{\|}=$ $\left(k^{0}+\mu, k^{1}, 0\right)$. The last term of the above equation is the responsible for the effect we are proposing and is independent of the gauge choice if we deal with an extended $(3+1)$-dimensional theory with one compactified dimension.

As it is well-known, some care must be taken when dealing with a chemical potential using the proper time method in a uniform magnetic background. ${ }^{20}$ The reason is that when the chemical potential is larger than the fermion mass, the propagator must be regularized in a certain $\mu$-dependent way. The time-ordered regulator is defined setting $k^{0} \rightarrow k^{0}(1+\varepsilon)$. As a consequence, in the denominator of Eq. (5), $K_{\|}^{2} \rightarrow K_{\|}^{2}+i \varepsilon 2 k^{0} K_{\|}^{0}$. So, in order to express the denominator as an integral of an exponential term, the convergence will be determined by the sign of $k^{0}\left(k^{0}+\mu\right)$. This can be written in a simple way as

$$
\frac{1}{i H_{ \pm}+\varepsilon 2 k^{0} K_{\|}^{0}}=\int_{-\infty}^{\infty} d s r_{s}\left(k^{0} K_{\|}^{0}\right) e^{-i s H_{ \pm}},
$$

where the regulation function, defined as

$$
r_{s}(\zeta)=\theta(s) \theta(\zeta) e^{-s \varepsilon}-\theta(-s) \theta(-\zeta) e^{s \varepsilon},
$$

ensures the correct convergence of the integral in the proper time. Now, the Schwinger proper-time method ${ }^{21}$ can be performed in the usual way by identifying the $y$-coordinate states as $\langle y|=\langle y(s)|$ and $\left|y^{\prime}\right\rangle=\left|y^{\prime}(0)\right\rangle$. We write the resulting chiral Green functions in an appropriate form:

$$
G_{ \pm}\left(r, r^{\prime}\right)=\int \frac{d^{3} k}{(2 \pi)^{3}} e^{-i k \cdot\left(r-r^{\prime}\right)} \tilde{G}\left(k ; \frac{1}{2}\left(y+y^{\prime}\right) e B+m_{ \pm}\right)
$$


where

$$
\begin{aligned}
\tilde{G}(k ; \xi) & =\int_{-\infty}^{\infty} d s r_{s}\left(k^{0} K_{\|}^{0}\right) e^{i s K_{\|}{ }^{2}-i\left[k_{2}{ }^{2}+\xi^{2}\right] \tan (e B s) / e B} \\
& \left\{K_{\|}\left[1+\gamma^{2} \gamma^{3} \tan (e B s)\right]+\left[k_{2} \gamma^{2}+\xi \gamma^{3}\right] \sec ^{2}(e B s)\right\} .
\end{aligned}
$$

The term $\xi=\frac{1}{2} e B\left(y+y^{\prime}\right)+m_{ \pm}$is a nonlocal factor along the direction perpendicular to the magnetic field on the plane.

The effects of a thermal bath are introduced by the replacements $k^{0} \rightarrow i \omega_{n}$, and $\int d k^{0} \rightarrow 2 \pi i T \sum_{n}$, where $\omega_{n}=(2 n+1) \pi T$ are the Matsubara frequencies, and the time component $t \rightarrow-i \tau$ is now compactified in the region $0 \leq \tau \leq 1 / T$ . The regulator for the proper-time can be introduced with the same analysis and the replacement is

$$
r_{s}\left(k^{0}\left(k^{0}+\mu\right)\right) \rightarrow r_{s}\left(\omega_{n} \mu\right),
$$

In the case of zero temperature in Euclidean space, the procedure follows the same line: $k^{0} \rightarrow-i k_{4}$ introducing the regulator in $r_{s}\left(-k_{4} \mu\right)$.

\section{Current densities}

Let us now calculate the currents. A current density is defined as

$$
(-e)\langle\bar{\psi} \Gamma \psi\rangle=e \operatorname{tr} \Gamma G(r, r)=j_{\Gamma}(y)
$$

where the trace is taken over spin and pseudospin indexes and where the operator $\Gamma$ can be $\gamma^{\mu}, \gamma^{\mu} \gamma^{5}, \gamma^{3}, \gamma^{3} \gamma^{5}$. The dependence on $y$, explicitly indicated in Eq. (12), appears due to the nonlocal term in the chiral Green function in Eq. (9) and (10).

Our model, even in the absence of a magnetic field, presents some non-vanishing expectation values: the particle number density $n=\left\langle\psi^{\dagger} \psi\right\rangle$ due to the chemical potential; the condensates $\sigma_{3}=\left\langle\bar{\psi} \gamma^{3} \psi\right\rangle$ and $\sigma_{o}=\left\langle\bar{\psi} \gamma^{3} \gamma^{5} \psi\right\rangle$ generated through the masses $m_{3}$ and $m_{o}$, respectively; and the chiral number density $n_{5}=\left\langle\psi^{\dagger} \gamma^{5} \psi\right\rangle$, due to the combination of chemical potential and masses. The presence of the uniform external magnetic field along the $x$-direction catalyzes the generation of two other currents along the same direction: an electric current $j_{x}=-e\left\langle\bar{\psi} \gamma^{1} \psi\right\rangle$ and an axial current $j_{5 x}=-e\left\langle\bar{\psi} \gamma^{1} \gamma^{5} \psi\right\rangle$, which is expected in analogy with the CME produced in QCD. We explore these currents in more detail below.

Following the expressions for the Green function in Eq. (3), (9) and (10), tracing over spin and pseudospin, we can write the electric induced current density and the axial current density as

$$
\begin{aligned}
j_{x}(y) & =j\left(y-y_{+}\right)-j\left(y-y_{-}\right), \\
j_{5 x}(y) & =j\left(y-y_{+}\right)+j\left(y-y_{-}\right),
\end{aligned}
$$

with $y_{ \pm}=-m_{ \pm} / e B$, and where the function $j$ reads

$$
j(\eta)=2 \frac{e^{2} B T}{2 \pi} \sum_{n} \int_{-\infty}^{\infty} d s r_{s}\left(\omega_{n} \mu\right)\left(\omega_{n}-i \mu\right)\left[\frac{\tan (e B s)}{e B s}\right]^{1 / 2}
$$




$$
\exp \left(-i\left[s\left(\omega_{n}-i \mu\right)^{2}+e B \tan (e B s) \eta^{2}\right]\right) .
$$

Note that the role of the spin in this case is simply to duplicate the degrees of freedom and it is not involved in any interaction.

It is possible to rotate the proper time integral in the complex plane at finite temperature. Following the restrictions imposed by the regulator $r_{s}$ defined in Eq. (8) and (11), we separate the proper time integral into the negative and positive ranges of integration. If $\omega_{n}^{2}>\mu^{2}$, the integrals can be enclosing the contour in the lower complex plane. If $\omega_{n}^{2}<\mu^{2}$, the contour of integration encloses in the upper complex plane. Both results are different, so, if we want a single expression for all the Matsubara frequencies, we must restrict ourselves to the condition $\pi T>\mu$. The function $j$ in this case can be written as

$$
\begin{gathered}
j(\eta)= \\
i \frac{e^{2} B T}{\pi} \sum_{n} \int_{0}^{\infty} d s\left(\omega_{n}-i \mu\right)\left[\frac{\tanh (e B s)}{e B s}\right]^{1 / 2} \\
\exp \left(-\left[s\left(\omega_{n}-i \mu\right)^{2}+e B \tanh (e B s) \eta^{2}\right]\right) .
\end{gathered}
$$

The integral above is highly suppressed by the exponential term $e^{-s\left(\omega_{n}^{2}-\mu^{2}\right)}$, where the range $s<(\pi T)^{2}-\mu^{2}$ dominates. If $|e B|<(\pi T)^{2}-\mu^{2}$, the product $e B s$ is too small in the relevant integration region. Then, we can make the approximation $\tanh (e B s) \approx e B s$ simplifying enormously (16), allowing us to integrate the proper time. After summing all the Matsubara frequencies we get this simple result:

$$
j(\eta) \approx-\frac{e^{2} B}{2 \pi}\left[n_{F}(|e B \eta|-\mu)-n_{F}(|e B \eta|+\mu)\right],
$$

being $n_{F}$ the Fermi-Dirac distribution.

The total electric current along the $x$ direction, following 22 , is then

$$
I_{x}=\int_{-L_{y} / 2}^{L_{y} / 2} d y\left[j\left(y-y_{+}\right)-j\left(y-y_{-}\right)\right],
$$

being $L_{y}$ the size of the plane in the $y$ direction.

\section{Conclusions}

In conclusion, we present here a new transport mechanism for systems represented by $(2+1)$-dimensional quantum electrodynamics in the presence of an external inplane uniform magnetic field. The systems considered present topological deformations represented by a CS term which induces a mass term proportional to $\gamma^{3} \gamma^{5}$ as well as sub-lattice deformations represented by a mass gap proportional to $\gamma^{3}$. We showed that the presence of an external in-plane magnetic field in such configuration generates an electric current along the field lines. Such a phenomenon can be regarded as an analogue of the CME proposed for QCD.

On one hand, the PCME has potential technological applications on material physics. On the other hand, because heavy ion collisions environment possess intrinsic ambiguities on the observables related to the non-trivial vacuum of $\mathrm{QCD}$, 
the condensed matter systems offer a more controlled environment that can provide valuable insight on the knowledge of the $\mathrm{QCD}$ vacua. Our expectations are that phenomena like the one we describe can shed light on the issue.

\section{Acknowledgements}

The Huitzil collaboration acknowledges J. A. Helayel-Neto for valuable discussions, W. Bietenholz for carefully reading this manuscript. We also acknowledge hospitality of UMSNH (Mexico) and UBB (Chile), where parts of this work were carried out, and La Porfiriana for the inspiration. AJM acknowledges CONACyT-Mexico under grant number 128534 and SECITI/CLAF under grant 023/2014. AR acknowledges support from CIC-UMSNH grant No. 4.22 and CONACyT-Mexico under grant number 128534. CV acknowledges support from FONDECYT under grant numbers 1150847,1130056 and 1150471.

\section{References}

1. Gusynin V P and Sharapov S G 2005 Phys. Rev. Lett. 95 146801;

Novoselov K S, Geim A K, Morozov S V, Jiang D, Katsnelson M I, Grigorieva I V, Dubonos S V and Firsov A A 2005 Nature (London) 438 197;

Zhang Y, Tan Y-W, Stormer H L and Kim P 2005 Nature (London) 438201.

2. Gusynin V P, Sharapov S G and Carbotte J P 2007 Int. J. Mod. Phys. B 214611.

3. Hasan M Z and Kane C L 2010 Rev. Mod. Phys. 82 3045;

Qi X and Zhang S 2011 Rev. Mod. Phys. 831057.

4. Franz M, Tesanovic Z, and Vafek O 2002 Phys. Rev. B 66 054535;

Herbut I F 2002 Phys. Rev. B 66 094504;

Thomas I O and Hands S 2007 Phys. Rev. B 75 134516;

Bonnet J A, Fischer C S and Williams R 2011 Phys. Rev. B 84024520.

5. Semenoff G W 1984 Phys. Rev. Lett. 53 2449;

Sharapov S G, Gusynin V P and Beck H 2004 Phys. Rev. B 69075104

6. Pisarski R D 1984 Phys. Rev. D 292423

7. Appelquist T, Bowick M J, Karabali D and Wijewardhana L C R 1986 Phys. Rev. D 33 3704;

Pennington M R and Walsh D 1991 Phys. Lett. B 253 246;

Kondo K-I and Maris P 1995 Phys. Rev. D 52 1212;

Hands S J, Kogut J B, and Strouthos C G 2002 Nucl. Phys. B 645 321;

Bashir A, Huet A, and Raya A 2002 Phys. Rev. D 66 025029;

Fischer C S, Alkofer R, Dahm T and Maris P 2004 Phys. Rev. D 70 073007;

Bashir A, Raya A, Clöet I and Roberts C D 2008 Phys. Rev. C 78 055201;

Hofmann C P, Raya A and Sánchez-Madrigal S 2010 Phys. Rev. D 82 096011;

Lo P M and Swanson E S 2011 Phys. Rev. D 83 065006;

Hofmann C P, Raya A, and Sánchez-Madrigal S 2011 J. Phys. Conf. Ser. 287012028.

8. Chern S S and Simons J 1974 Ann. Math. 9948.

9. Khare A, Fractional Statistics and Quantum Theory 2005 (World Scientic, Singapore).

10. Kharzeev D E, McLerran L D and Warringa H J 2008 Nucl. Phys. A 803227 ;

Fukushima K, Kharzeev D E and Warringa H J 2008 Phys. Rev. D 85045104.

11. Cocco G, Cadelano E and Colombo L 2010 Phys. Rev. B 81 241412(R);

Guinea F, Katsnelson M I and Geim A K 2010 Nature Phys. 6 30;

Woods C R et al. 2014 Nature Phys. 10451. 
12. Ryu S, Mudry C, Hou C-Y and Chamon C 2009 Phys. Rev. B 80205319.

13. Giovannetti G, Khomyakov P A, Brocks G, Kelly P J and Brink Jeroen 2007 Phys. Rev. B 76073103.

14. Castro-Neto A H, Guinea F, Peres N M R, Novoselov K S, and Geim A K 2009, Rev. Mod. Phys. 81 109;

Guinea F, Katsnelson M I, and Geim A K 2010, Nature Phys. 6 30;

Guinea F, Geim A K, Katsnelson M I, and Novoselov K S 2010, Phys. Rev. B 81 035408;

Levy N, Burke S A, Meaker K L, Panlasigui M, Zettl A, Guinea F, Castro-Neto A H, and Crommie M F 2010, Science 329 544;

de Juan F, Mañes J L, and M. A. H. Vozmediano 2013, M A H, Phys. Rev. B 87 $165131 ;$

Peña F J and Muñoz E 2015, Phys. Rev. E 91 052152;

Sadurní E, Rivera-Mociños E and Rosado A 2015, Rev. Mex. Fis. 61170.

15. Zubkov M A 2015, Annals Phys. 360655.

16. Coleman S and Hill B 1985 Phys. Lett. B 159 184;

Delbourgo R and Waites A 1994 Aust. J. Phys. 47 465;

Raya A and Reyes E D 2008 J. Phys. A 41355401.

17. Kotikov A V and Teber S 2014 Phys. Rev. D 89065038.

18. Abreu E M C, Helayel-Neto J A, Hott M and Moura-Melo W A 2002 Phys. Rev. D 65085024.

19. Andersen J O 1996, Phys. Rev. D 53, 7286.

20. Chodos A, Everding K and Owen D A 1990 Phys. Rev. D 422881.

21. Schwinger J 1951 Phys. Rev. D 82664.

22. Miransky V A and Shovkovy I A 2015, Phys. Rept. 5761 